\title{
Encalado y micorriza para corregir deficiencia de fósforo en un Andisol cultivado con Pinus halepensis
}

\section{Lime and mycorrhizae in the phosphorus deficiencies correction in an andisol cultivated with Pinus halepensis Mill.}

\author{
María E. Álvarez-Sanchez ${ }^{1}$, Elizabeth Hernández-Acosta ${ }^{1^{*}}$, \\ Ranferi Maldonado-Torres ${ }^{1}$ y Miguel Rivera-González ${ }^{2}$
}

\begin{abstract}
RESUMEN
Con la finalidad de evaluar el encalado del suelo y el potencial de inoculación de una multicepa con propágulos de hongos micorrícicos arbuscular (HMA) en el desarrollo y acumulación de fósforo de Pinus halepensis Mill., cultivado en un Andisol, en invernadero se evaluó la aplicación de cal para elevar el pH del suelo de 5,3 a 5,5; 6,0 y 6,5; con y sin la adición de micorriza. Los resultados mostraron que la inoculación micorrícica o el encalado del suelo para alcanzar un pH de 6,0 a 6,5; promovieron el mayor desarrollo de los brinzales medido como peso seco aéreo, diámetro del tallo, altura y volumen radical, así como en la acumulación de fósforo por las plantas, en comparación con el testigo (sin cal agrícola o sin inocular con HMA). El encalado estimuló el porcentaje de colonización, independientemente de la inoculación con HMA. La validación en campo del tratamiento de encalado a pH de 6,5 e inoculación con HMA promovió $95 \%$ de supervivencia de los árboles con un incremento promedio en altura y diámetro de tallo de $22,1 \mathrm{~cm}$ y $5 \mathrm{~cm}$, respectivamente, durante un período de evaluación de cinco meses. Los resultados de invernadero y campo sugieren la conveniencia de encalar e inocular con micorriza arbuscular como prácticas efectivas para el establecimiento comercial de $P$. halepensis en Andisoles del estado de México.
\end{abstract}

PALABRAS CLAVE:

Carbonato de calcio, inoculación micorrícica arbuscular, Pinus halepensis Mill.

\begin{abstract}
In order to evaluate soil liming and the potential of inoculation of a multistrain with propagules of arbuscular mycorrhizal fungi (AMF) in the development and accumulation of $P$. halepensis Mill phosphorus, grown in an Andisol, it was evaluated, in a greenhouse, the application of lime to raise the soil pH from 5,3 to 5,$5 ; 6,0$ and 6,5 ; with and without the addition of mycorrhizae. The results showed that mycorrhizal inoculation and the soil liming to $\mathrm{pH}$ of 6,0 to 6,5 ; led to a further development of seedling, measured in dry aerial weight, stem diameter, height, and root volume, as well as phosphorus accumulation by plants, compared with the control (with no agricultural liming and without inoculation with AMF). Liming stimulated the percentage of colonization, regardless inoculation with AMF. The field validation of the liming treatment to $\mathrm{pH} 6,5$ and $\mathrm{AMF}$ inoculation promoted $95 \%$ survival of trees with an average increase in height and stem diameter of $22,1 \mathrm{~cm}$ and $5 \mathrm{~cm}$, respectively, during an evaluation period of five months. The greenhouse and field results suggest the benefits of liming and inoculation with arbus-
\end{abstract} tera México-Texcoco, Texoco, Méx.56230. C.e.: edna_alvarez30@yahoo.com.mx

2 Exestudiante de posgrado. Universidad Autónoma Chapingo. km 38.5 carretera México-Texcoco, Texoco, Méx. 56230

* Autor para correspondencia: elizahac@yahoo.com 
cular mycorrhizal as effective practices for the commercial establishment of $P$. halepensis in Andisols in the state of Mexico.

KEY WORDS:

lime, arbuscular mhycorrizae, Pinus halepensis Mill, Andisoil, phosphorus.

\section{INTRODUCCIÓN}

En el oriente del estado de México existen bosques de clima templado con predominio de especies de cipreses, encinos y pinos. Los suelos donde se desarrolla esta vegetación son principalmente Andisoles, cuyas características distintivas son $\mathrm{pH}$ ácido y alto contenido de alofano, material amorfo que retiene el fósforo disponible y lo hace muy poco aprovechable para las plantas (Shoji et al., 1993). La baja disponibilidad de fósforo en estos suelos y el exceso de aluminio activo limitan el desarrollo y la producción de las plantaciones forestales. La reforestación y el aprovechamiento comercial de $P$. halepensis en Andisoles del estado de México como alternativa de manejo en estos ecosistemas alterados, requiere en el corto plazo después del trasplante, una rápida adaptación que maximice la supervivencia de los árboles; para esto, es necesario generar condiciones óptimas de nutrición para el desarrollo de dicha especie forestal, entre otros factores. El encalado de los suelos es una de las prácticas que se emplean para corregir deficiencias de fósforo, reducir los niveles de toxicidad del aluminio y favorecer la disponibilidad de nutrimentos como el calcio, magnesio y molibdeno (Alvarado y Fallas, 2004); también se recurre a la inoculación con micorrizas para aumentar la eficiencia en el aprovechamiento de los nutrimentos del suelo, en especial de fósforo.

Hasta hace pocos años, se consideraba que especies de la familia Pinaceae sólo desarrollaban simbiosis con los hon- gos ectomicorrícicos, sin embargo, trabajos realizados por Smith et al. (1998) y Cameron et al. (2011) demuestran que especies de la familia Pinaceae, como $P$. contorta, $P$. ponderosa y $P$. strobus, forman simbiosis con hongo micorrícico arbuscular (HMA) de la especie Glomus intraradices. La inoculación con HMA en algunas especies maderables en vivero ha mostrado algunos resultados prometedores en la supervivencia de los árboles en programas de restauración de comunidades tropicales (Ramos-Zapata y Guadarrama, 2004) y paisajismo (Appleton et al., 2003; Wiseman y Wells, 2005).

La importancia ecológica de la colonización con HMA en especies vegetales típicamente ectomicorrícicos es escasa y amerita determinar si esta relación favorece la nutrición, desarrollo y supervivencia de plántulas de pinos para su producción comercial.

\section{OBJETIVO}

Evaluar el encalado de un Andisol y el potencial de la inoculación con micorriza arbuscular en el desarrollo y acumulación de fósforo de brinzales de pino $P$. halepensis Mill. y validar en campo el mejor tratamiento en una plantación con dicha especie.

\section{METODOLOGÍA}

\section{Experimento en invernadero}

Se empleó un suelo con $\mathrm{pH}$ moderadamente ácido $(5,3)$ medido en agua, un contenido bajo de fósforo disponible determinado por el método P-Bray $(5 \mathrm{mg}$ $\mathrm{kg}^{-1}$ ), según las metodologías indicadas en la Norma Oficial Mexicana NOM-021-RECNAT-2000 (Se-marnat, 2002). Con base en sus características, este suelo es clasificado como Andisol. 
Se probaron dosis de $0 \mathrm{~g} \mathrm{~kg}^{-1} ; 0,2 \mathrm{~g} \mathrm{~kg}^{-1}$; $1,0 \mathrm{~g} \mathrm{~kg}^{-1}$ y $2,0 \mathrm{~g} \mathrm{~kg}^{-1}$ de suelo de cal agrícola $\left(\mathrm{CaCO}_{3}\right)$ con $90 \%$ de pureza, para incrementar el $\mathrm{pH}$ de 5,3 a 5,$5 ; 6,0$ y 6,5 ; con y sin inoculación micorrícica. La dosis de cal aplicada en cada tratamiento se estimó de acuerdo con la técnica de la curva de titulación descrita por Goijberg y Aguilar (1987), para alcanzar el pH deseado. El inóculo micorrícico comercial conocido como Endospor formado por las especies Gigaspora margarita, Glomus mosseae, Glomus clarum, Glomus deserticola, Glomus etunicatum, Glomus brasilianum y Glomus intraradices, se aplicó a una dosis de $5 \mathrm{~g}$ por brinzal, a través del agua de riego en el momento de la plantación.

El experimento correspondió a un factorial con el primer factor dado por el encalado (con cuatro niveles) y el segundo factor inoculación micorricica con dos niveles (sin y con inoculación); su combinación generó ocho tratamientos. Dadas las condiciones de invernadero, se empleó un diseño experimental completamente al azar con cinco repeticiones. La unidad experimental estuvo constituida por un recipiente que contenía un brinzal.

La dosis de cal se aplicó al suelo 15 días antes del trasplante, tiempo suficiente para lograr que se estabilizara el $\mathrm{pH}$ al valor propuesto; posteriormente, los brinzales de $P$. halepensis Mill. de $41 \mathrm{~cm}$ (desviación estándar $(d s)= \pm 3,0 \mathrm{~cm}$ ) de altura promedio se colocaron en bolsas de polietileno negro con capacidad para $5 \mathrm{~kg}$ de suelo. El experimento tuvo una duración de cinco meses.

\section{Medición de variables de crecimiento}

Al final del experimento, en cada uno de los brinzales, se midió la altura total de planta desde la base hasta el ápice y el diámetro del tallo en la base. Una vez extraído y lavado el sistema radical, se determinó su volumen en una probeta a partir del nivel de agua desplazada. Se colectaron muestras de raíz para evaluar la colonización micorrícica; el resto del material vegetal se secó en estufa de aire forzado a $70^{\circ} \mathrm{C}$ y se registró el peso seco, luego se midió la concentración de fósforo en el tejido de la parte aérea en un digerido (mezcla de $\mathrm{HClO}_{4}$ y $\mathrm{HNO}_{3}$ concentrado, relación 1:2), mediante el método del fosfovanadomolibdato (Jackson, 1970). También se tomaron muestras de suelo de cada unidad experimental, para determinar pH (en agua, relación 1:2) y P-Bray (Semarnap, 2002).

Para evaluar la colonización micorrícica, se tiñeron raíces finas por el método de Phillips y Hayman (1970); el porcentaje de longitud radical colonizada se determinó por el método de las intersecciones. También se realizaron cortes histológicos y tinción en parafina para observar la presencia de las estructuras micorrícicas (hifas, esporas y arbúsculos).

Las variables de respuesta evaluadas se sometieron a un análisis de varianza acorde con el diseño de tratamientos y experimental empleados (factorial completamente al azar) y a una prueba de medias entre niveles de encalado e inoculación micorrícica según el procedimiento de Tukey $(\alpha=0,05)$ para las variables diámetro de tallo, peso seco aéreo, fósforo acumulado en planta, volumen de raíz y segmentos colonizados, en donde la interacción no resultó significativa.

\section{Experimento en campo}

El experimento de campo consistió en evaluar el mejor tratamiento obtenido en el invernadero, que fue adición de cal agrícola para alcanzar un $\mathrm{pH}$ de 6,5 e inoculación micorrícica. Éste se instaló en el predio forestal El Llano, ubicado en el ejido San Miguel Coatlinchán, Texcoco, 
México, en las coordenadas geográficas $19^{\circ} 24^{\prime} 20^{\prime \prime}$ y $19^{\circ} 29^{\prime} 30^{\prime \prime}$ latitud norte y $98^{\circ} 47^{\prime} 00^{\prime \prime}$ y $98^{\circ} 29^{\prime} 30^{\prime \prime}$ longitud oeste. El suelo del predio presentó a una profundidad de $0 \mathrm{~cm}$ a $30 \mathrm{~cm}$, $\mathrm{pH}$ de 5,$3 ; 6,0 \%$ de materia orgánica; $5 \mathrm{mg} \mathrm{kg}^{-1}$ de P-Bray-1; $6,2 \mathrm{cmol} \mathrm{kg}^{-1} ; 1,8 \mathrm{cmol} \mathrm{kg}^{-1}$ y $0,9 \mathrm{cmol} \mathrm{kg}^{-1}$ de $\mathrm{Ca}$, Mg y K intercambiables; $31,0 \mathrm{mg} \mathrm{kg}^{-1}$, $1,0 \mathrm{mg} \mathrm{kg}^{-1}, 16,2 \mathrm{mg} \mathrm{kg}^{-1}$ y $0,5 \mathrm{mg} \mathrm{kg}^{-1} \mathrm{de}$ $\mathrm{Fe}, \mathrm{Cu}, \mathrm{Mn}, \mathrm{Zn}$ y $\mathrm{B}$; y $42 \%$ de saturación de aluminio, según las metodologías indicadas en la Norma Oficial Mexicana NOM-021-RECNAT-2000 (Semarnap, 2002).

El experimento de campo consistió en la siembra de 40 árboles de $P$. halepensis, distribuidos a un espaciamiento promedio entre árboles de $2,5 \mathrm{~m}$ por $1,8 \mathrm{~m}$. El encalado se realizó 60 días antes del trasplante, las plantas se inocularon con la micorriza y se procedió a la plantación de 20 árboles con altura inicial promedio de 41,8 $\mathrm{cm}$ (desviación estándar $(d s)= \pm 3,5 \mathrm{~cm}$ ) y un diámetro de tallo de $5,5 \mathrm{~cm}(d s= \pm 0,8$ $\mathrm{cm}$ ), el resto de la población correspondió al tratamiento testigo (sin cal y sin inoculación con micorriza) que presentaba una altura de $49 \mathrm{~cm}(d s= \pm 5,6 \mathrm{~cm})$ y un diá- metro de $5,4 \mathrm{~cm}(d s= \pm 0,7 \mathrm{~cm})$. Se evaluó altura de planta y diámetro del tallo, al inicio de la plantación y después de cinco meses de su establecimiento en campo, también se cuantificó la supervivencia de brinzales.

\section{RESULTADOS Y DISCUSIÓN}

\section{Experimento de invernadero}

El encalado afectó positiva y significativamente el desarrollo de los brinzales en las variables altura, diámetro del tallo y volumen de raíz; la acumulación de fósforo en la planta y el porcentaje de segmentos de raíz colonizados. La inoculación con HMA influenció significativamente a todas las variables de crecimiento; $P$ acumulado en la planta y segmentos de raíz colonizados. La interacción de estos factores sólo fue significativamente positiva en la altura de planta. Por otra parte, tanto el encalado como la inoculación con HMA no influyeron en la disponibilidad de fósforo (P-Bray) en el suelo (Tabla 1).

Tabla 1. Pruebas de significancia de los factores niveles de encalado (E), micorriza (HMA) y su interacción en las variables de crecimiento, fósforo acumulado y segmentos de raíz colonizados en brinzales de pino.

\begin{tabular}{|c|c|c|c|c|c|c|c|}
\hline $\begin{array}{l}\text { Fuentes de } \\
\text { variación }\end{array}$ & Altura & Diámetro tallo & $\begin{array}{c}\text { Peso } \\
\text { seco aéreo }\end{array}$ & $\begin{array}{l}\text { Volumen de } \\
\text { raíz }\end{array}$ & $\begin{array}{c}P \\
\text { acumulado }\end{array}$ & $\begin{array}{c}\text { Segmentos } \\
\text { de raíz colonizados }\end{array}$ & P-Bray \\
\hline & \multicolumn{2}{|c|}{------- cm ------- } & g planta $^{-1}$ & $\mathrm{~cm}^{3}$ & mg planta-1 & $\%$ & $\mathrm{mg} \mathrm{kg}^{-1}$ \\
\hline Encalado & ** & ** & ns & ** & $* *$ & ** & ns \\
\hline HMA & $* *$ & ** & ** & ** & ** & * & ns \\
\hline $\mathrm{E} \times \mathrm{HMA}$ & ** & ns & ns & ns & ns & ns & ns \\
\hline${ }^{z} \mathrm{CV}(\%)$ & 6,2 & 8,5 & 10,7 & 14,9 & 18,8 & 12,5 & 20,9 \\
\hline & ificat & & & ** & ificativo a & $<0,01$ & \\
\hline & cativo a & 0,05 & & ${ }^{\mathrm{z}} \mathrm{CV}$ & Deficiente de & ariación & \\
\hline
\end{tabular}


En la figura 1 se muestra la interacción de los factores estudiados en altura de planta. Los niveles de encalado que elevaron el $\mathrm{pH}$ del suelo de 5,3 a 6,0 y 6,5 (niveles 2 y 3 , respectivamente), favorecieron significativamente el crecimiento en altura de los brinzales con respecto a los tratamientos sin encalar y con o sin inocular con HMA; aunque sin diferencias significativas entre estos niveles. Con el nivel de encalado 3 (equivalente a pH 6,5) e inoculación con HMA, el incremento en altura fue de $48 \%$ con respecto al tratamiento sin encalar y sin inocular, y de 30,1\% con inoculación micorrícica. En la tabla 2 se presenta el efecto del encalado y el de la inoculación micorrícica en las variables donde no se manifestó la interacción de estos factores. El aumento en el $\mathrm{pH}$ del suelo con el nivel 3 de encalado también favoreció significativamente el desarrollo de los árboles en términos de diámetro y volumen de raíz, con incrementos de 48,6\% y $53,8 \%$, respectivamente. Asimismo, la adición de micorrizas contribuyó en el crecimiento de los brinzales, aunque sólo resultó en un incremento de $9,3 \%$ en el diámetro del tallo y de $38,5 \%$ en el volumen radical. A diferencia de estas variables de crecimiento, el peso seco de la planta sólo fue influenciado con la inoculación HMA, con un incremento significativo de $36,2 \%$, con respecto al promedio de los tratamientos sin inocular.

El encalado y la inoculación con HMA no ejercieron un efecto significativo en el fósforo disponible en el suelo (P-Bray), éste se incrementó de $5 \mathrm{mg} \mathrm{kg}^{-1}$ a $5,9 \mathrm{mg}$ $\mathrm{kg}^{-1}$, contenido considerado como deficiente en el suelo (Castellanos, 2000). Sin embargo, tanto la adición de micorrizas como el encalado del suelo se reflejaron en la nutrición con fósforo de los brinzales. En la tabla 2 se muestra que tanto el aumento en el $\mathrm{pH}$ del suelo a 6 (nivel 2 de encalado) como la adición de micorriza favorecieron el fósforo acumulado en la planta, con un incremento de $55,9 \%$ y de $31,1 \%$, en comparación con el promedio de los tratamientos sin encalar o sin inocular, respectivamente. Los niveles de fósforo extractable no necesariamente reflejan la disponibilidad de fós-

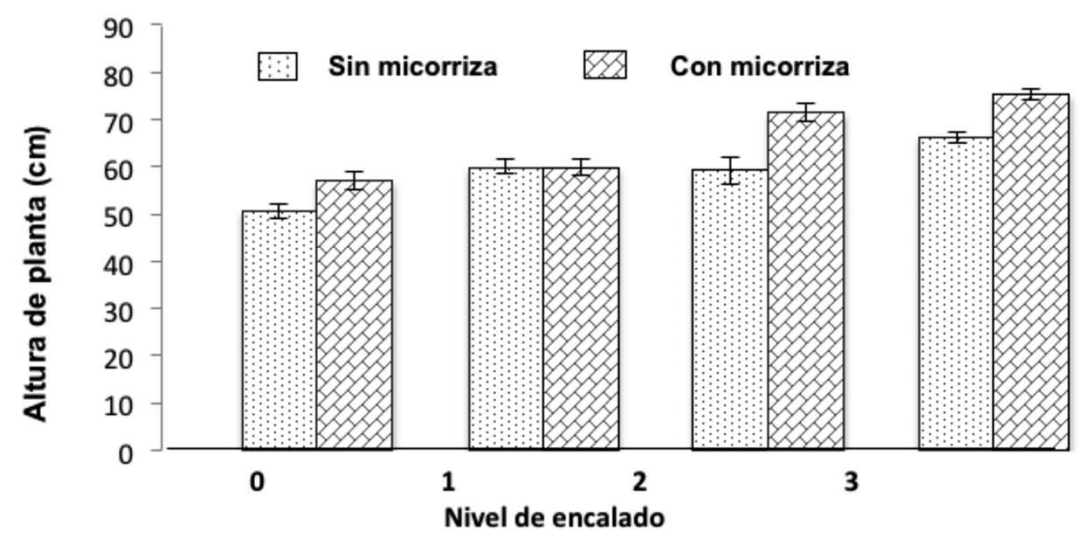

Figura 1. Efecto de la interacción nivel de encalado e inoculación micorrícica en la altura de planta de brinzales de pino ( $P$. halepensis Mill.). 
foro para las plantas, ya que las hifas de los hongos pueden mejorar el acceso a este nutrimento por las raíces, debido a su gran capacidad de explorar entre los poros más finos del suelo, donde las raíces de la planta no pueden penetrar (Smith y Read, 2008); de incrementar la actividad de la rizósfera a través de la liberación de fosfatasas que mineralizan el fósforo orgánico (Feng et al., 2002) y de mantener tasas de influjo de fosfato hacia la superficie radical superiores al de las raíces de la planta (Smith et al., 2001). Estos efectos pudieron ocurrir en los brinzales de $P$. halepensis, ya que fue notable el incremento en el volumen radical generado por la micorriza $(38,5 \%)$, con respecto al tratamiento sin encalar o sin inocular, lo que demuestra el efecto benéfico de aumentar la densidad radical y con ello la capacidad de exploración de las raíces en el suelo y, por tanto, de acceso a los nutrimentos.

Si bien no hubo interacción entre los factores estudiados en la mayoría de las variables evaluadas, la inoculación con HMA ejerció un efecto sinérgico favorable para el desarrollo de $P$. halepensis.

Existen pocos estudios del efecto de la inoculación con HMA en pináceas o especies maderables que son típicamente ectomicorrícicas, las respuestas en el crecimiento de las plantas pueden ir desde sin efecto (Smith et al., 1998; Appleton et al., 2003), con algún efecto favorable Morrison et al., 1993) o incluso en detrimento del crecimiento (Cameron et al., 2011).

Entre los factores que pueden influir en la falta de respuesta a la inoculación con HMA están: niveles de alta fertilidad (Morrison et al., 1993) y de fósforo disponible en el suelo (Wiseman y Wells, 2005), el género dentro de la especie de micorriza empleada (Smith, 1998; Cameron, 2011) combinada con una adecuada fertilización de las plantas (Appleton et al., 2003).

\section{Colonización micorrícica}

Las observaciones realizadas al microscopio permitieron identificar hifas y esporas, pero no arbúsculos. Estas estructuras se han identificado en raíces de pináceas inoculadas con especies de las familias Glomaceae (Smith et al., 1998; Cameron et al., 2011). En la tabla 2 se observa que las raíces del tratamiento sin encalar, así como las del tratamiento sin inocular estuvieron altamente colonizadas por HMA (31,1\% y $46,9 \%$, respectivamente). Diferentes investigaciones muestran que un suelo forestal (o sustratos compuestos con material vegetal) puede contener una importante proporción de propágulos de HMA (Morrison et al., 1993; Appleton et al., 2003; Wiseman y Wells, 2005), dicho potencial de inoculación depende de un nivel deficiente en fósforo extractable (Montaño et al., 2001; Wiseman y Wells, 2005) y que el suelo contenga fuentes naturales de propágulos derivados de plantas hospederas de HMA, aunque la especie de planta hospedera puede, a su vez, definir el desarrollo de los HMA (Carrenho et al., 2002).

El aumento del pH del suelo influyó significativamente en el desarrollo de la colonización por hifas y vesículas en las raíces de los brinzales de pino. $\mathrm{A}$ un $\mathrm{pH}$ de 6,5 el porcentaje de colonización fue de $70,2 \%$ y de $55,7 \%$ con la inoculación; en ambos casos, fue notable el incremento en el porcentaje de segmentos colonizados con respecto al promedio de los tratamientos sin encalar $(31,1 \%)$ o sin inocular (46,9\%). La importancia de los resultados obtenidos en este estudio radican en que el incremento en los niveles de colonización alcanzados -aun con competencia de micorrizas autóctonas- estuvieron asociados de forma significativa en el corto tiempo (cinco meses) con el aumento en el crecimiento y nutrición con fósforo de $P$. halepensis. Diferentes investigaciones realizadas en árboles madera- 
Tabla 2. Efecto del encalado (medias sobre los tratamientos de inoculación micorrícica) y de la inoculación micorrícica (medias sobre los tratamientos de encalado) en las variables evaluadas.

\begin{tabular}{|c|c|c|c|c|c|c|}
\hline Factor & $\begin{array}{c}\text { Diámetro } \\
\text { tallo }\end{array}$ & $\begin{array}{c}\text { Peso } \\
\text { seco aéreo }\end{array}$ & $\begin{array}{l}\text { Volumen de } \\
\quad \text { raíz }\end{array}$ & $\begin{array}{c}P \\
\text { acumulado }\end{array}$ & $\begin{array}{c}\text { Segmentos } \\
\text { de raíz } \\
\text { colonizados }\end{array}$ & P-Bray \\
\hline & $\mathrm{cm}$ & g planta ${ }^{-1}$ & $\mathrm{~cm}^{3}$ & mg planta ${ }^{-1}$ & $\%$ & $\mathrm{mg} \mathrm{kg}^{-1}$ \\
\hline \multicolumn{7}{|l|}{$\begin{array}{l}\text { Niveles de } \\
\text { encalado }\end{array}$} \\
\hline 0 & $7,4 \mathrm{c}$ & $27,8 a$ & $26 c$ & $44,7 \mathrm{~b}$ & $31,1 \mathrm{c}$ & 5,4 \\
\hline 1 & $7,7 \mathrm{c}$ & $29,5 a$ & $25 c$ & $57,1 \mathrm{ab}$ & $52,3 \mathrm{~b}$ & 5,7 \\
\hline 2 & $9,8 b$ & $30,5 a$ & $33,0 \mathrm{~b}$ & $69,7 a$ & $51,6 \mathrm{~b}$ & 6,1 \\
\hline 3 & $11,0 a$ & $28,4 a$ & $40,0 a$ & $60,4 a$ & $70,2 a$ & 6,4 \\
\hline 'DMS1 & 0,93 & 3,78 & 5,58 & 13,22 & 14,53 & ns \\
\hline Con HMA & $9,4 a$ & $33,5 a$ & $36,0 a$ & $65,8 a$ & $55,7 a$ & 5,9 \\
\hline Sin HMA & $8,6 \mathrm{~b}$ & $24,6 \mathrm{~b}$ & $26,0 \mathrm{~b}$ & $50,2 b$ & $46,9 b$ & 5,8 \\
\hline ¿DMS2 & 0,49 & 2,01 & 2,97 & 7,00 & 7,40 & ns \\
\hline
\end{tabular}

¿DMS1 para comparar entre niveles de encalado,

DMS2 para comparar entre niveles de inoculación micorrícica, según prueba de Tukey $(\alpha=0,05)$.

Letras iguales dentro de una columna según el factor de comparación, no son significativamente distintas.

bles inoculados con HMA, con frecuencia, no han mostrado beneficios tangibles en el crecimiento de los árboles, aunque hayan desarrollado un alto porcentaje de colonización; en otros casos, el beneficio de la inoculación se ha presentado años después. Por ejemplo, Morrison et al. (1993) inocularon con Glomus intraradices o Glomus fasciculatum a las especies maderables Acer platanoide L., Sorbus aucuparia L., Malus Mill. y Fraxinus pennsylvanica March.; la inoculación incrementó significativamente la colonización en dichas especies, pero sin efecto en el crecimiento. Después de la inoculación, las especies se trasplantaron a un segundo campo con competencia de hongos micorrícicos nativos; dos años después del postrasplante, sólo Glomus intraradices mejoró en forma significativa el crecimiento de $S$. aucuparia. Appleton et al. (2003) utilizaron un producto comercial con micorrizas (los géneros predominantes fueron Glomus clarum, Glomus etunicatum, Glomus intraradices y Entrophospora columbiana) en encino (Quercus palustris y Quercus phellos) y maple (Acer rubrum), después de un año los árboles no mostraron respuesta a la inoculación medida por el contenido de cloro- 
fila o diámetro del tronco; sin embargo, cuando se hizo una combinación de inóculo comercial con fertilizante $(9 \%$ de $\mathrm{N}$, $45 \% \mathrm{P}_{2} \mathrm{O}_{5}$ y $15 \%$ de $\mathrm{K}_{2} 0$ ) el peso seco de las raíces del encino y del maple se incrementó significativamente así como el porcentaje de colonización. Smith et al. (1998) inocularon plántulas de diferentes especies de pináceas con Glomus intraradices $\mathrm{y}$, aunque las plántulas presentaron colonización después de ocho semanas de desarrollo, no hubo efectos significativos en el peso seco aéreo y de raíz.

Por otra parte, estudios realizados por Cameron et al. (2011) indican que el efecto de la inoculación con HMA en el crecimiento de plántulas de pino está relacionado con la familia a la que pertenecen los HMA. Ellos probaron dos especies de HMA de las familias Glomaceae (G. intraradices y $G$ mosseae), Acaulosporaceae (Entrophospora columbiana y Acaulospora morrowiae) y Gigasporineae (Gigaspora margarita y Scutellospora calospora) en su capacidad para colonizar y afectar el crecimiento de pino blanco (Pinus strobus).

Después de siete semanas de desarrollo, Glomus intraradices fue el único que colonizó las raíces de los pinos; las especies Acaulosporaceae redujeron la biomasa de las plántulas, mientras que las de Gigasporineae tuvieron el menor efecto sobre el peso seco. El producto comercial que se utilizó en la presente investigación contenía una multicepa compuesta por especies de la familia Glomaceae (Glomus mosseae, G. clarum, G. deserticola, G. etunicatum, G. brasilianum, G. intraradices) y una especie de Gigasporineae (Gigaspora margarita), por lo tanto, la compatibilidad de las especies de HMA contenidas en el inóculo comercial, el nivel deficiente de fósforo extractable en el suelo y la práctica del encalado contribuyeron a la respuesta significativa en el desarrollo y nutrición del $P$. halepensis.

\section{Validación en campo}

La validación en campo, en un período de evaluación de cinco meses, con el tratamiento de encalado a $\mathrm{pH}$ de 6,5 y micorriza promovió $95 \%$ de supervivencia de los árboles, con un incremento promedio en altura de $22,1 \mathrm{~cm}(\mathrm{sd}= \pm 6,9 \mathrm{~cm})$ y diámetro de tallo de $5 \mathrm{~cm}$ ( $\mathrm{sd}= \pm 1,2 \mathrm{~cm}$ ), en comparación con el testigo, en el que sólo hubo $10 \%$ de supervivencia. El incremento en la supervivencia de las plantas micorrizadas podría explicarse por una mejora del estado nutrimental estimulada por el encalado, en el momento de la plantación, en relación con las plantas no inoculadas. Los resultados de este experimento en el período de evaluación (cinco meses) demuestran que la inoculación de los brinzales del pino en el momento del trasplante, mejoró la supervivencia y el crecimiento de esta especie en el Andisol donde se establecieron los pinos.

La mayoría de los trabajos de investigación sobre reforestación con coníferas inoculadas con hongos ectomicorrícicos han mostrado, desde hace 60 años, que mejora los procesos de repoblación y revegetación en distintas situaciones ambientales y de suelos (Pera y Parladé, 2005). Estos antecedentes no existen para coníferas inoculadas con hongos endomicorrícicos. Las interacciones de especies de hongos micorrícicos arbusculares con pináceas son recientes (Cameron et al., 2011).

\section{CONCLUSIONES}

Los resultados de invernadero y campo sugieren la conveniencia del encalado e inoculación con endomicorrizas, como una práctica con posibilidades de efectividad para la producción comercial de $P$. halepensis en la zona de estudio, debido al incremento significativo en altura, diámetro de tallo, volumen radical colonización micorrícica, fósforo acumulado en los brinzales y de supervivencia en campo. 


\section{BIBLIOGRAFÍA}

Alvarado, A. y J.L. Fallas. 2004. La saturación de acidez y el encalado sobre el crecimiento de la teca (Tectona grandis L F) en suelos ácidos de Costa Rica. Agronomía Costarricense 28(1):81-87.

Appleton, B., J. Koci, S. French, M. Lestyan y R. Harris. 2003. Mycorrhizal fungal inoculation of established street trees. Journal of Arboriculture 29(2):107-110.

Cameron, W., A.P. Madeira y L.R. Peterson. 2011. Arbuscular mycorrhizal fungal phylogeny-related interactions with a non-host. Symbiosis 53(1)41-46.

Carrenho, R., S.F.B. Trufem y V.L.R. Bononi. 2002. Effects of using different host plants on the detected biodiversity of arbuscular mycorrhizal fungi from agroecosystem. Revista Brasileira de Botânica 25(1):93-101.

Castellanos R., J.Z., J.X. Uvalle-Bueno y A. Aguilar-Santelises. 2000. Manual de interpretación de análisis de suelos y aguas. $2^{a}$ ed. Instituto de Capacitación para la Productividad Agrícola. San Miguel de Allende, Guanajuato. 226 p.

Feng, G.Y., Y. Su, X. Li, H. Wang, F. Zhang, C. Tang y Z. Rengel. 2002. Histochemical visualization of phosphatase released by arbuscular mycorrhizal fungi in soil. Journal of Plant Nutrition 25(5):969-980.

Goijberg, G. y A. Aguilar. 1987. pH del suelo y necesidades de cal. In: S.A. Aguilar, J. D. Etchevers B., J. Z. Castellanos R., eds. Análisis químico para evaluar la fertilidad del suelo. Sociedad Mexicana de la Ciencia del Suelo. Publicación Especial núm. 1. Chapingo, Estado de México. p:17-44.
Jackson, M.L. 1970. Análisis químico de suelos. Ed. Omega, S.A. Barcelona, España. 662 p.

Montaño A., M.N., G.V. Quiroz y F.G. Cruz. 2001. Colonización micorrízica arbuscular y fertilización mineral de genotipos de maíz y trigo cultivados en un Andisol. Terra Latinoamericana 19(4):337-344.

Morrison, S.J., P.A. Nicholl y P.R. Hicklenton. 1993. VA mycorrhizal inoculation of landscape trees and shrubs growing under high fertility conditions. Journal of Environmental Horticulture 11(2):64-71.

Pera, J. y J. Parladé. 2005. Inoculación controlada con hongos ectomicorrízicos en la producción de planta destinada a repoblaciones forestales: estado actual en España. Investigación agraria: Sistemas y recursos forestales 14(3):419-433.

Phillips, J.M. y D.S. Hayman. 1970. Improved procedures for clearing roots for rapid assessment of infection. Transactions of the British Mycological Society 55:(1)158-161.

Ramos-Zapata, J. y P. Guadarrama. 2004. Los hongos micorrizógenos arbusculares en la restauración de comunidades tropicales. Universidad y Ciencia, Número Especial l:59-65.

Semarnap. 2002. Norma Oficial Mexicana NOM-021-RECNAT-2000, que establece las especificaciones de fertilidad, salinidad y clasificación de suelos, estudio muestreo y análisis. Secretaria de Medio Ambiente, Recursos Naturales y Pesca. Diario Oficial, martes 31 de diciembre de 2002. Segunda Sección. México DF. $85 \mathrm{p}$. 
Shoji, S., R. Dahlgren y M. Nanzyo. 1993. Volcanic ash Soils. Genesis, properties and utilization. Elsevier. Amsterdam Londres - Nueva York - Tokyo. 275 p.

Smith, J.E., K.A. Johnson y E. Cázares. 1998. Vesicular mycorrhizal colonization of seedlings of Pinaceae and Betulaceae after spore inoculation with Glomus intraradices. Mycorrhiza 7(6):279-285.

Smith, S.E., S. Dickson y E.A. Smith. 2001. Nutrient transfer in arbuscular mycorrhizas: How are fungal and plant processes integrated? Australian Journal of Plant Physiology 28(7):683-694.

Smith, S.E. y D.J. Read. 2008. Mycorrhizal Symbiosis. Academic Press. Nueva York, EUA. 787 p.

Wiseman, P. E. y Ch. Wells. 2005. Soil inoculum potential and arbuscular mycorrhizal colonization of Acer rubrum in forested and developed landscapes. Journal of Arboriculture 31(6):296-302. 